\title{
End-Stage Renal Disease Patients on Chronic Hemodialysis Fare Better With COVID-19: A Retrospective Cohort Study From the New York Metropolitan Region
}

Ashutossh Naaraayan ${ }^{1}$, Abhishek Nimkar ${ }^{1}$, Amrah Hasan ${ }^{1}$, Sushil Pant ${ }^{1}$, Momcilo Durdevic ${ }^{1}$, Henrik Elenius ${ }^{1}$, Corina Nava Suarez ${ }^{1}$, Prasanta Basak ${ }^{1}$, Kameswari Lakshmi ${ }^{2}$, Michael Mandel ${ }^{2}$, Stephen Jesmajian ${ }^{1}$

1. Internal Medicine, Montefiore New Rochelle Hospital, Albert Einstein College of Medicine, New Rochelle, USA 2. Internal Medicine, Montefiore New Rochelle Hospital, New Rochelle, USA

Corresponding author: Ashutossh Naaraayan, ashu.newroc@gmail.com

\section{Abstract}

\section{Introduction}

Several comorbid conditions have been identified as risk factors in patients with coronavirus disease 2019 (COVID-19). However, there is a dearth of data describing the impact of COVID-19 infection in patients with end-stage renal disease on hemodialysis (ESRD-HD).

\section{Methods}

This retrospective case series analyzed 362 adult patients consecutively hospitalized with confirmed COVID19 illness between March 12, 2020, and May 13, 2020, at a teaching hospital in the New York City metropolitan area. The primary outcome was severe pneumonia as defined by the World Health Organization. Secondary outcomes were the (1) the Combined Outcome of Acute respiratory distress syndrome or in-hospital Death (COAD), and (2) need for high levels of oxygen supplementation $\left(\mathrm{HiO}_{2}\right)$.

\section{Results}

Patients with ESRD-HD had lower odds for poor outcomes including severe pneumonia [odds ratio (OR) 0.4, confidence interval (CI) 0.2-0.9, p=.04], $\mathrm{HiO}_{2}$ [OR 0.3, CI (0.1-0.8), p=.02] and COAD [OR 0.4, CI (0.2-1.05), $\mathrm{p}=.06]$, when compared to patients without ESRD. In contrast, higher odds for severe pneumonia, COAD and $\mathrm{HiO}_{2}$ were seen with advancing age. African Americans were over-represented in the hospitalized patient cohort, when compared to their representation in the community (35\% vs $18 \%$ ). Hispanics had higher odds for severe illness and $\mathrm{HiO}_{2}$ when compared to Caucasians.

Received 08/24/2020

Review began 08/25/2020 Review ended 09/01/2020 Published 09/11/2020

() Copyright 2020

Naaraayan et al. This is an open access article distributed under the terms of the Creative Commons Attribution License CC-BY 4.0., which permits unrestricted use, distribution, and reproduction in any medium, provided the original author and source are credited.

\section{Conclusions}

Patients with ESRD-HD had a milder course of illness with a lower likelihood of severe pneumonia and a lesser need for aggressive oxygen supplementation when compared to patients not on chronic dialysis. The lower odds of severe illness in ESRD-HD patients might have a pathophysiologic basis and need to be further explored.

Categories: Infectious Disease, Nephrology, Epidemiology/Public Health

Keywords: coronavirus disease 2019 (covid-19), maintenance hemodialysis, end stage renal disease (esrd), better outcomes, covid-19 pneumonia, oxygen support, acute respiratory distress syndrome [ards], absolute mortality, racial disparity

\section{Introduction}

At the time of writing, 25 million cases of coronavirus disease 2019 (COVID-19) caused by severe acute respiratory syndrome coronavirus-2 (SARS-CoV-2) have been described worldwide, resulting in 850,000 deaths [1]. COVID-19 was declared a pandemic by the World Health Organization (WHO) on March 11, 2020, and the New York City (NYC) metropolitan region was at its epicenter through March and April, 2020. So far, laboratory-confirmed cases have been documented from 188 countries on six continents, with the United States reporting the highest number of cases and deaths [1].

It is crucial to identify the demographic and comorbid factors that impact illness severity among hospitalized COVID-19 patients. Several comorbid conditions, such as hypertension, diabetes, chronic obstructive pulmonary disease and atherosclerotic cardiovascular diseases, have been identified as risk factors in patients with COVID-19 [2,3]. However, there is a dearth of data describing the impact of COVID- 
19 infection in patients with end-stage renal disease on hemodialysis (ESRD-HD).

Being on the frontlines at one of the earliest epicenters in the United States, we observed that patients with ESRD-HD were having a milder course of illness compared to patients not on HD. Here, we present data analyzing the impact of patient characteristics on illness severity among hospitalized COVID-19 patients, while specifically investigating the effect of ESRD-HD on outcomes. (Paper:

Ashutossh Naaraayan, Abhishek Nimkar, Amrah Hasan, et al. Chronic Hemodialysis Patients Have Better Outcomes With COVID-19 - A Retrospective Cohort Study; July 24, 2020)

\section{Materials And Methods \\ Data source}

This retrospective observational study includes patients consecutively hospitalized with confirmed COVID19 illness between March 10, 2020, and May 13, 2020, at a 242-bed teaching community hospital in the NYC metropolitan region. Cases were confirmed through positive results for SARS-CoV-2 virus by reversetranscriptase polymerase-chain-reaction testing of the nasopharyngeal swab specimen. Data was manually abstracted from electronic health records by the authors, and included demographics, comorbid conditions and clinical data (respiratory rate, oxygen saturations, arterial blood gas tests when available, type/level of oxygen supplementation). Three authors (AN, AN and SP) independently reviewed the data for accuracy.

Hospital course was followed up until June 15, 2020. Details on the criteria for hospitalization are provided in the supplementary material in the Appendix. Of the 377 patients admitted during the study period, those who were transferred to another facility for tertiary level care $(n=5)$, those who left against medical advice $(n=2)$ and those who were still receiving care at our hospital $(n=8)$ were excluded from the analysis. After said exclusions, final analysis included 362 patients.

\section{Definition of patient characteristics}

Comorbidities derived from the patients or nursing home transfer forms were abstracted from physician documentation on the electronic health records. Patients were classified into three categories based on the presence or absence of kidney disease: (i) no chronic kidney disease, (ii) presence of chronic kidney disease and (iii) presence of ESRD-HD. Outcomes were analyzed in patients with chronic kidney disease versus those without kidney disease and patients with ESRD-HD versus those without kidney disease. Cardiac disease was defined as chronic heart conditions including previous myocardial infarction, cardiac arrhythmias, congestive heart failure, presence of pacemaker or defibrillator device and previous coronary artery bypass grafting or percutaneous coronary intervention. Body mass index $>=30 \mathrm{~kg} / \mathrm{m}^{2}$ was used to identify obesity. Race/ethnicity was classified into one of the four categories: Caucasian, African American, Hispanic ethnicity regardless of race, and others.

Severe disease was defined as clinical pneumonia with a respiratory rate $>30$ breaths/minute or oxygen saturation $<90 \%$ on room air, per the WHO guidelines [4]. Acute respiratory distress syndrome (ARDS) was defined as the ratio of arterial oxygen partial pressure to fractional inspired oxygen of $<=300$ on a positive end-expiratory pressure $>=5 \mathrm{~cm}$ of water, as per the Berlin guidelines [5]. Mortality was defined as inhospital death. Need for aggressive oxygen supplementation with high-flow nasal cannula, non-invasive positive pressure ventilation or mechanical ventilation was defined as high-oxygen support $\left(\mathrm{HiO}_{2}\right)$.

\section{Outcome measures and statistical analysis}

The study was done according to the STrengthening the Reporting of OBservational studies in Epidemiology (STROBE) guidelines for observational studies [6]. We computed median with inter-quartile range, frequency, and percentages as our descriptive variables. Differences in median were calculated using the Mann-Whitney test. Differences in percentage were assessed using the chi-squared test. Our primary outcome was the development of severe pneumonia as defined by the WHO. Our secondary outcomes were the (1) need for $\mathrm{HiO}_{2}$ ), and (2) Combined Outcome of a diagnosis of ARDS or in-hospital Death (COAD).

Logistic regression analysis by univariate and age-adjusted models was performed for patient characteristics. Subsequently, to determine independent association between these characteristics and outcomes, multivariable logistic regression models were used to estimate odds ratios (ORs) adjusted for clinical covariates. Multivariable analysis was performed using the least absolute shrinkage and selection operator (Lasso) inference, a standard model building approach [7]. Lasso is most useful for sparse high-dimensional models such as ours. Double selection was used for estimation as this inference method provides the most stable results. Stata version 16.0 (StataCorp, Houston, TX) was used for analysis. Age, sex, hypertension, diabetes and cardiac disease were always included for adjustment in this model. Lasso was asked to select variables in the model from the following specified covariates: race, chronic obstructive pulmonary disease, renal disease and obesity. Each variable of interest was fitted separately for multivariable analysis. When one of these covariates was the variable of interest, it was not specified to Lasso for model selection. Overfitting and collinearity were resolved by using Lasso and defining variables as stated above. Two-sided $\mathrm{p}<.05$ was considered statistically significant. 


\section{Cureus}

\section{Ethics}

The study was approved by the departmental research review committee with a waiver of informed consent due to its retrospective design (approval number 20.5.02).

\section{Results}

Among the 362 patients, 55.3\% were men, 35.4\% were African American and 232 (63\%) had severe disease; the median age was 71 years (59-82) (Table 1). There were no deaths among patients without severe disease. The length of hospital stay was longer among patients with severe disease. Patients with severe disease were more likely to be Hispanic and less likely to have ESRD-HD. Twelve of 27 (44.4\%) of ESRD patients had severe disease as opposed to 232/335 (69.3\%) of patients not on chronic $\mathrm{HD}(\mathrm{p}=.008)$.

\begin{tabular}{|c|c|c|c|c|}
\hline Characteristic & Entire cohort $(n=362)$ & Non-severe disease $(n=130)$ & Severe disease $(n=232)$ & p-value \\
\hline Age, years - median (IQR) & $71(59-82)$ & $70(58-78)$ & $73(60-84.5)$ & .1 \\
\hline Sex: male & $200(55.3)$ & $68(52.3)$ & 132 (56.9) & .4 \\
\hline \multicolumn{5}{|l|}{ Race } \\
\hline Caucasian & $123(33.9)$ & $43(33.1)$ & 80 (34.5) & .8 \\
\hline African American & $128(35.4)$ & $49(37.7)$ & $79(34.1)$ & .5 \\
\hline Hispanic & $71(19.6)$ & 18 (13.9) & $53(22.9)$ & .04 \\
\hline Others & 40 (11.1) & 20 (15.4) & $20(8.6)$ & .05 \\
\hline \multicolumn{5}{|l|}{ Comorbidities } \\
\hline Hypertension & $241(66.6)$ & $85(65.4)$ & $156(67.2)$ & .7 \\
\hline Diabetes & $154(42.5)$ & $50(38.5)$ & $104(44.8)$ & .2 \\
\hline Cardiac diseases & 119 (32.9) & $42(32.3)$ & 77 (33.2) & .9 \\
\hline \multicolumn{5}{|l|}{ Renal disease } \\
\hline CKD & $41(11.3)$ & $12(9.2)$ & 29 (12.5) & .4 \\
\hline ESRD-HD & $27(7.5)$ & 15 (11.5) & $12(5.2)$ & .03 \\
\hline COPD & 50 (13.8) & $21(16.2)$ & 29 (12.5) & .3 \\
\hline Obesity & $123(33.9)$ & 41 (31.5) & 82 (35.4) & .5 \\
\hline LOS median (IQR) & $6(4-10)$ & $5(3-7)$ & $8(5-12.5)$ & \\
\hline Mortality, n (\%) & $149(41.2)$ & $0(0)$ & (64.2) & \\
\hline
\end{tabular}

\section{TABLE 1: Baseline characteristics of hospitalized COVID-19 patients}

CKD: chronic kidney disease; COPD: chronic obstructive pulmonary disease, ESRD-HD: end-stage renal disease on hemodialysis; IQR: interquartile range; LOS: length of hospital stay; COVID-19: coronavirus disease 2019

Data are presented as number (percentage) except for age and length of stay.

Patients with ESRD-HD had lower odds of severe pneumonia on univariate analysis compared to patients without kidney disease (Table 2). 


\section{Cureus}

\begin{tabular}{|c|c|c|}
\hline & OR (Cl) & $p$-value \\
\hline Age (1-year increments) & $1.01(0.9-1.03)$ & .08 \\
\hline Male sex (ref. female) & $1.2(0.8-1.9)$ & .4 \\
\hline \multicolumn{3}{|l|}{ Race (ref. Caucasian) } \\
\hline African American & $0.9(0.5-1.5)$ & .6 \\
\hline Hispanic & $1.6(0.8-3.03)$ & .2 \\
\hline Others & $0.5(0.3-1.1)$ & .1 \\
\hline \multicolumn{3}{|l|}{ Comorbidities } \\
\hline Hypertension & $1.09(0.7-1.7)$ & .7 \\
\hline Diabetes & $1.3(0.8-2.01)$ & .2 \\
\hline Cardiac diseases & $1.04(0.7-1.7)$ & .9 \\
\hline \multicolumn{3}{|l|}{ RD (ref. no RD) } \\
\hline CKD & $1.3(0.6-2.7)$ & .5 \\
\hline ESRD-HD & $0.4(0.2-0.9)$ & .04 \\
\hline COPD & $0.7(0.4-1.4)$ & .3 \\
\hline Obesity & $1.2(0.8-1.9)$ & .5 \\
\hline
\end{tabular}

\section{TABLE 2: Univariate analysis of patient characteristics for severe pneumonia}

$\mathrm{Cl}$ : confidence interval; OR: odds ratio; CKD: chronic kidney disease; COPD: chronic obstructive pulmonary disease, ESRD-HD: end-stage renal disease on hemodialysis; RD: renal disease; ref.: reference

Hispanics had higher age-adjusted odds of severe disease while patients with ESRD-HD had lower odds for the same (Table 3). On multivariable logistic regression, advancing age and Hispanic ethnicity had higher odds of severe disease/pneumonia. In contrast, patients with ESRD-HD [OR 0.4 (0.2-0.9), p=.04] demonstrated lower odds of severe disease compared to patients without kidney disease (Table 3, Figure 1). 


\section{Cureus}

\begin{tabular}{|c|c|c|c|c|}
\hline & Age-adjusted OR (CI) & p-value & Multivariable OR (Cl) & $p$-value \\
\hline Age (1-year increments) & - & - & $1.03(1.01-1.04)$ & .006 \\
\hline Male sex (ref. female) & $1.3(0.8-2.04)$ & .2 & $1.3(0.8-2.02)$ & .3 \\
\hline \multicolumn{5}{|l|}{ Race (ref. Caucasian) } \\
\hline African American & $0.9(0.6-1.7)$ & .9 & $0.9(0.6-1.7)$ & .9 \\
\hline Hispanic & $2.2(1.1-4.5)$ & .03 & $2.2(1.1-4.7)$ & .04 \\
\hline Others & $0.6(0.3-1.2)$ & .2 & $0.6(0.3-1.3)$ & .2 \\
\hline \multicolumn{5}{|l|}{ Comorbidities } \\
\hline Hypertension & $0.9(0.6-1.5)$ & .8 & $0.9(0.6-1.7)$ & .9 \\
\hline Diabetes & $1.3(0.4-2.01)$ & .3 & $1.4(0.9-2.2)$ & .2 \\
\hline Cardiac diseases & $0.9(0.6-1.5)$ & .7 & $0.9(0.5-1.4)$ & 6 \\
\hline \multicolumn{5}{|l|}{ RD (ref. no RD) } \\
\hline CKD & $1.2(0.6-2.4)$ & .7 & $1.1(0.5-2.3)$ & .8 \\
\hline ESRD-HD & $0.4(0.2-0.9)$ & .04 & $0.4(0.2-0.9)$ & .04 \\
\hline COPD & $0.7(0.4-1.2)$ & .2 & $0.7(0.4-1.2)$ & .2 \\
\hline Obesity & $1.4(0.9-2.2)$ & .2 & $1.3(0.8-2.2)$ & .3 \\
\hline
\end{tabular}

\section{TABLE 3: Risk factors for severe pneumonia}

$\mathrm{Cl}$ : confidence interval; CKD: chronic kidney disease; COPD: chronic obstructive pulmonary disease; ESRD-HD: end-stage renal disease on hemodialysis; OR: odds ratio; RD: renal disease; ref.: reference.

p-values for the multivariable models were derived after always adjusting for age, sex, hypertension, diabetes and cardiac disease. Lasso was asked to select variables in the model from the following specified covariates: race, chronic obstructive pulmonary disease, renal disease and obesity). If the covariate itself was the variable of interest, it was not included in the adjustment model.

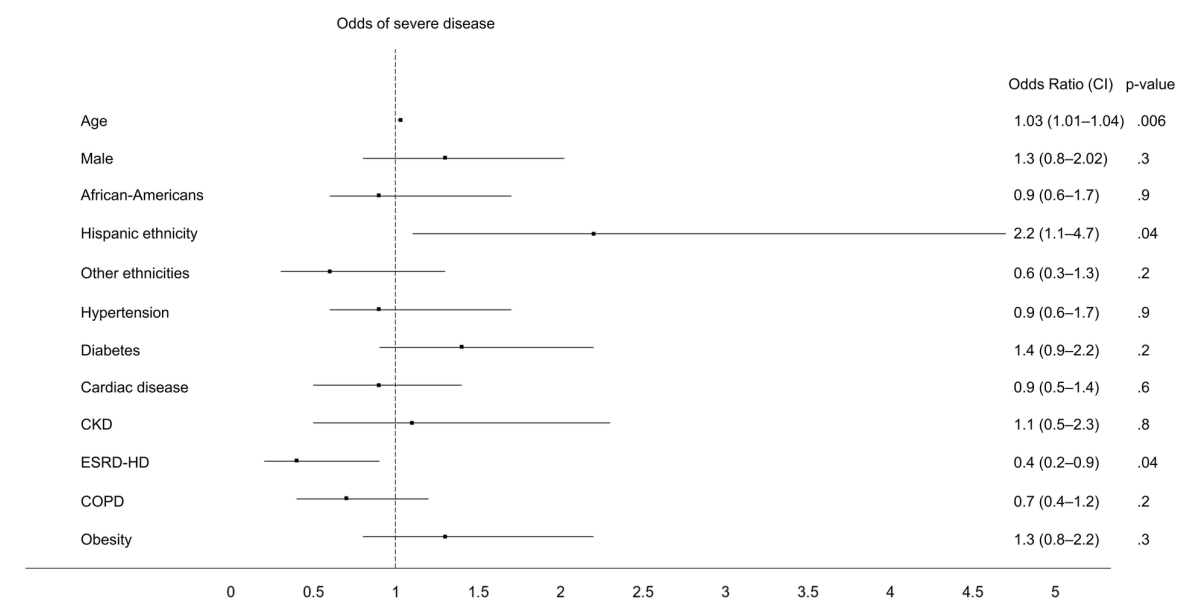

FIGURE 1: Forest plot showing multivariable odds of severe disease

ARDS: acute respiratory distress syndrome; CKD: chronic kidney disease; COPD: chronic obstructive pulmonary disease; ESRD-HD: end-stage renal disease on hemodialysis; Cl: confidence interval

Additionally, advancing age showed higher odds while ESRD demonstrated lower odds for the secondary outcomes, $\mathrm{HiO}_{2}$ and COAD (Tables 4, 5, Figures 2, 3). 


\section{Cureus}

\begin{tabular}{|c|c|c|c|c|}
\hline & Age-adjusted OR (Cl) & p-value & Multivariable OR (CI) & p-value \\
\hline Age (1-year increments) & - & - & $1.02(1.003-1.04)$ & .02 \\
\hline Male sex (ref. female) & $0.9(0.6-1.4)$ & 0.8 & $0.9(0.6-1.4)$ & .7 \\
\hline \multicolumn{5}{|l|}{ Race (ref. Caucasian) } \\
\hline African American & $0.9(0.6-1.6)$ & .8 & $0.8(0.5-1.4)$ & .5 \\
\hline Hispanic & $1.9(0.9-3.6)$ & .06 & $1.9(0.9-3.8)$ & .056 \\
\hline Others & $0.8(0.4-1.8)$ & .6 & $0.7(0.3-1.5)$ & .4 \\
\hline \multicolumn{5}{|l|}{ Comorbidities } \\
\hline Hypertension & $1.3(0.8-2.2)$ & .2 & $1.4(0.9-2.4)$ & .2 \\
\hline Diabetes & $1.5(0.9-2.3)$ & .06 & $1.5(0.9-2.4)$ & .08 \\
\hline Cardiac diseases & $0.8(0.5-1.4)$ & .5 & $0.8(0.5-1.3)$ & .3 \\
\hline \multicolumn{5}{|l|}{ RD (ref. no RD) } \\
\hline CKD & $0.7(0.4-1.4)$ & 0.4 & $0.6(0.3-1.2)$ & .2 \\
\hline ESRD-HD & $0.3(0.1-0.9)$ & .03 & $0.3(0.1-0.8)$ & .02 \\
\hline COPD & $1.06(0.6-1.9)$ & .9 & $1.1(0.6-1.9)$ & .8 \\
\hline Obesity & $1.4(0.9-2.3)$ & .1 & $1.3(0.8-2$. & .2 \\
\hline
\end{tabular}

\section{TABLE 4: Risk factors for high-oxygen requirement}

$\mathrm{Cl}$ : confidence interval; CKD: chronic kidney disease; COPD: chronic obstructive pulmonary disease; ESRD-HD: end-stage renal disease on hemodialysis; OR: odds ratio; RD: renal disease; ref.: reference.

p-values for the multivariable models were derived after always adjusting for age, sex, hypertension, diabetes and cardiac disease. Lasso was asked to select variables in the model from the following specified covariates: race, chronic obstructive pulmonary disease, renal disease and obesity. If the covariate itself was the variable of interest, it was not included in the adjustment model. 


\section{Cureus}

\begin{tabular}{|c|c|c|c|c|}
\hline & Age-adjusted OR (Cl) & p-value & Multivariable OR (Cl) & p-value \\
\hline Age (1-year increments) & & & $1.05(1.03-1.07)$ & \\
\hline Male sex (ref. female) & $1.04(0.7-1.6)$ & .2 & $1.02(0.7-1.6)$ & .9 \\
\hline \multicolumn{5}{|l|}{ Race (ref. Caucasian) } \\
\hline African American & $1.03(0.6-1.7)$ & .9 & $0.9(0.6-1.6)$ & .9 \\
\hline Hispanic & $1.7(0.9-3.2)$ & .1 & $1.7(0.9-3.3)$ & .1 \\
\hline Others & $0.6(0.3-1.4)$ & .3 & $0.6(0.3-1.2)$ & .2 \\
\hline \multicolumn{5}{|l|}{ Comorbidities } \\
\hline Hypertension & $1.1(0.7-1.8)$ & .6 & $1.1(0.7-1.9)$ & .6 \\
\hline Diabetes & $1.4(0.9-2.1)$ & $\begin{array}{llll}.1 & & \end{array}$ & $1.4(0.9-2.2)$ & .2 \\
\hline Cardiac diseases & $0.9(0.6-1.6)$ & .9 & $0.9(0.6-1.5)$ & .7 \\
\hline \multicolumn{5}{|l|}{ RD (ref. no RD) } \\
\hline CKD & $1.1(0.5-2.1)$ & .9 & $0.9(0.5-1.9)$ & .9 \\
\hline ESRD-HD & $0.5(0.2-1.1)$ & .087 & $0.4(0.2-1.05)$ & .06 \\
\hline COPD & $0.8(0.4-1.4)$ & .4 & $0.8(0.4-1.4)$ & .4 \\
\hline Obesity & $1.7(1.03-2.7)$ & .04 & $1.6(0.9-2.6)$ & .08 \\
\hline
\end{tabular}

\section{TABLE 5: Risk factors for combined outcome of ARDS or in-hospital death}

$\mathrm{Cl}$ : confidence interval; CKD: chronic kidney disease; COPD: chronic obstructive pulmonary disease; ESRD-HD: end-stage renal disease on hemodialysis; OR: odds ratio; RD: renal disease; ref.: reference

p-values for the multivariable models were derived after always adjusting for age, sex, hypertension, diabetes and cardiac disease. Lasso was asked to select variables in the model from the following specified covariates: race, chronic obstructive pulmonary disease, renal disease and obesity. If the covariate itself was the variable of interest, it was not included in the adjustment model. 


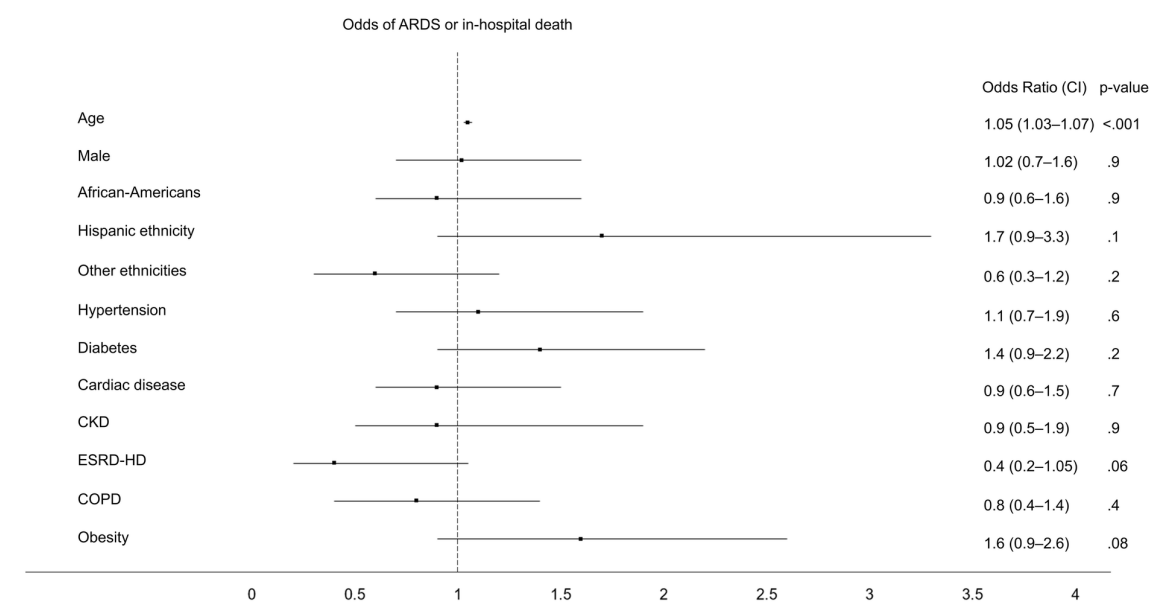

\section{FIGURE 3: Forest plot showing multivariable odds of the combined outcome of ARDS and in-hospital mortality}

ARDS: acute respiratory distress syndrome; CKD: chronic kidney disease; COPD: chronic obstructive pulmonary disease; ESRD-HD: end-stage renal disease on hemodialysis; CI: confidence interval

\section{Discussion}

In this study, we present data identifying patient characteristics that affect the illness severity of people hospitalized with COVID-19. The findings of decreased illness severity with ESRD in hospitalized COVID-19 patients are novel and need further investigation. No such association was seen in patients with chronic kidney disease not on HD. This was also observed firsthand by the authors by being on the frontlines in New Rochelle, New York, one of the first COVID-19 epicenters in the United States. Only one other group of researchers has described a lower severity of disease in dialysis patients with COVID-19 [8]. In that study, Ma et al. describe mostly mild clinical symptoms in patients with ESRD and a low likelihood of progression to severe pneumonia in ESRD-HD patients in an outpatient setting. To the best of our knowledge, our study is the first to demonstrate a lower illness severity in ESRD-HD patients in the inpatient setting. Of note, some studies have mentioned poor outcomes in ESRD-HD patients [9,10]. However, none of the studies to date have compared outcomes between ESRD-HD patients and patients not on hemodialysis, like we did in the present study.

Additionally, our study demonstrated higher illness severity and worse outcomes with advancing age. This is not surprising as by now, advancing age has been established as the most important risk factor for poor outcomes in COVID-19 [11,12]. Male sex and comorbidities (hypertension, diabetes, cardiac disease, obesity and others) have also been identified as independent risk factors for worse outcomes [11,12]. In our patient cohort, the odds for severe illness, $\mathrm{COAD}$ and $\mathrm{HiO}_{2}$ trended higher among diabetics and obese, although this did not satisfy our pre-determined requirement for statistical significance.

Patients belonging to ethnic and racial minorities are increasingly being identified to be at a higher risk with COVID-19 [13]. At first glance, it may seem that minorities were not particularly affected in our study; however, a deeper dive into the data would dispel that notion. As per the 2010 US census, the racial/ethnic makeup of New Rochelle was as follows: 45\% non-Hispanic Caucasians, 20\% African Americans and 28\% Hispanics of any race [14]. Thus, even though we did not see higher odds for poor outcomes among African American inpatients, they were over-represented among the hospitalized patients when compared to their representation in the community ( $35 \%$ vs $18 \%$ ). In addition, we found that patients of Hispanic ethnicity had higher odds for severe illness when compared to Caucasians.

Our finding begs the question, what are the pathophysiologic mechanisms behind this association between ESRD-HD and lower illness severity with COVID-19?

In the previous influenza and novel coronavirus epidemics, SARS (2003) and Middle East respiratory syndrome (2012), in addition to virus-induced cytopathic effects, an excessive and dysregulated host immune response played a crucial role in the pathology and mortality $[15,16]$. This exaggerated response with supraphysiologic levels of cytokines is referred to as cytokine release syndrome or "cytokine storm". Cytokine storm has been described as a major determinant of the severity of illness in COVID-19 patients as well $[17,18]$. Thus, during viral epidemics, illness severity and outcomes depend on the balance between the beneficial anti-viral effect of the immune response and the harm from an exaggerated inflammatory 
reaction.

ESRD-HD is simultaneously associated with immune activation leading to systemic inflammation and immune deficiency [19]. The activation of the constituents of the innate immune system, monocytes, macrophages, and granulocytes, is responsible for ESRD-associated inflammation. On the other hand, immune deficiency in ESRD is caused by the depletion of dendritic cells, memory T cells, B cells and impaired phagocytic function of neutrophils and monocytes [19]. Thus, one would expect ESRD-HD patients to have a poor antiviral response and an exaggerated inflammatory response with COVID-19, potentially manifesting as poor outcomes in these patients.

An immune-deficient state was indeed demonstrated by Ma et al. in their study [8]. A significantly lower number of T cells, natural killer cells, and B cells in ESRD-HD patients was seen compared with patients not on dialysis [8]. These numbers in ESRD-HD patients with SARS-CoV-2 infection were further decreased. However, paradoxically, the serum levels of inflammatory markers (interleukin-4, interleukin-6 and tumor necrosis factor-alpha) were highest in non-HD patients with COVID-19 infection, followed by non-HD patients without COVID-19, and the lowest levels of inflammatory markers were observed in ESRD-HD patients irrespective of COVID-19 infection.

Thus, on one hand, ESRD-HD patients were at a higher risk due to the decreased ability for an effective antiviral response from an immunodeficient state, and on the other hand, they benefited from a lower propensity for inflammatory tissue damage secondary to a dampened cytokine release and a lower likelihood of cytokine storm. Our observation of the lower likelihood of severe disease and lesser requirement for oxygen supplementation suggests that the balance between the benefit from anti-viral immune response and harm from associated inflammatory damage is tilted favorably for individuals with ESRD on HD.

This raises another question, as to why do ESRD-HD patients, who chronically demonstrate an inflammatory state [20-22], had significantly lower levels of inflammatory markers in COVID-19 infection compared to patients not on HD [8]? Perhaps, it is possible that "preconditioning" with this chronic underlying inflammation in ESRD-HD patients attenuates the inflammatory response from an acute insult such as the COVID-19 infection, thereby decreasing the inflammatory damage, illness severity and the need for supportive therapy. This hypothesis is analogous to the well-established "obesity paradox", in which preconditioning with chronic inflammation in obese patients is thought to contribute to a dampened inflammatory response leading to better outcomes in acute critical illness [23,24]. Additionally, there is a possibility that the inflammatory cytokines that are generated in response to the acute infection are removed by dialysis membranes during hemodialysis [25].

Another mechanism for better outcomes in chronic-dialysis patients could be related to the reninangiotensin system (RAS). It has been suggested that excessive activation of the RAS might contribute to the progression of COVID-19-related lung injury [26]. Chronic perturbations of the RAS and intra-renal RAS in ESRD-HD have been described [27,28]. Modifications in the activation of the RAS in response to the acute COVID-19 illness in patients with ESRD-HD might explain the "protective effect" seen in these patients.

The previously described phenomenon of "reverse epidemiology", the finding that conventional cardiovascular risk factors such as obesity, hypertension, and dyslipidemia are not associated with increased mortality in the hemodialysis population, also points to the fact that ESRD-HD patients are a distinct subgroup and do not respond to risks and insults the same way as general population [29,30]. From our observation and the findings from the study by Ma et al., it is likely that the altered immune milieu and physiology in patients with ESRD modifies the inflammatory (cytokine) response to acute COVID-19 illness in these patients, giving them an advantage in the short term. Our findings are novel and of great general medical interest, and should be shared, corroborated and elucidated.

\section{Strengths and limitations}

Limitations include a patient cohort that comprised only hospitalized patients, from a single hospital and included older individuals, and thus, the findings might not be generally applicable. We did not review the effect of medications targeting the RAS (acetyl cholinesterase inhibitors and angiotensin receptor blockers) on illness severity. Strengths of the study include the accuracy of data that was manually extracted from patient charts, a relatively large cohort and using a standardized model building technique (Lasso) for multivariable analysis.

\section{Conclusions}

Patients with ESRD-HD had a milder course of illness with lower likelihood of development of severe pneumonia and lower need for aggressive oxygen supplementation when compared to patients not on chronic dialysis. Our observations suggest that the balance between the benefit from an anti-viral immune response and harm from associated inflammatory damage is tilted favorably for individuals with ESRD-HD. The lower odds of severe illness in ESRD-HD patients might have a pathophysiologic basis and need to be further explored. Additionally, age had the greatest impact on illness severity, oxygen requirement and mortality in patients hospitalized with COVID-19. African Americans were over-represented among the 
hospitalized COVID-19 patients compared to the community, and Hispanics had a more severe illness course compared to Caucasians.

\section{Appendices \\ Supplementary material}

Criteria for hospital admission

a. Admit all patients with suspected COVID-19 and oxygen saturations $<90 \%$ by pulse oximetry.

b. Admit for oxygen saturations $91 \%-94 \%$ if

i. >=65 years age and comorbidities (diabetes, chronic lung, liver or kidney disease or on immunosuppressants)

ii. $<65$ years if considerable gastrointestinal losses

iii. Regardless of age if patient from a nursing home/congregate setting

c. Admit for oxygen saturation $>=95 \%$ if

i. Patient from a nursing home/shelter or similar congregate setting

\section{Additional Information \\ Disclosures}

Human subjects: Consent was obtained by all participants in this study. Department of Medicine Research Review Committee issued approval Approval number 20.5.02. The study was carried out in accordance with the Declaration of Helsinki, and was approved by the departmental research review committee with a waiver of informed consent due to its retrospective design (approval number 20.5.02). Animal subjects: All authors have confirmed that this study did not involve animal subjects or tissue. Conflicts of interest: In compliance with the ICMJE uniform disclosure form, all authors declare the following: Payment/services info: All authors have declared that no financial support was received from any organization for the submitted work. Financial relationships: All authors have declared that they have no financial relationships at present or within the previous three years with any organizations that might have an interest in the submitted work. Other relationships: All authors have declared that there are no other relationships or activities that could appear to have influenced the submitted work.

\section{References}

1. COVID-19 dashboard by the Center for Systems Science and Engineering (CSSE) at Johns Hopkins Coronavirus Resource Center. (2020). Accessed: September 1, 2020: https://coronavirus.jhu.edu/map.html.

2. Yang J, Zheng Y, Gou X, et al.: Prevalence of comorbidities and its effects in patients infected with SARSCoV-2: a systematic review and meta-analysis. Int J Infect Dis. 2020, 94:91-95. 10.1016/j.ijid.2020.03.017

3. Wang B, Li R, Lu Z, Huang Y: Does comorbidity increase the risk of patients with COVID-19: evidence from meta-analysis. Aging (Albany NY). 2020, 12:6049-6057. 10.18632/aging.103000

4. Clinical management of COVID-19: interim guidance. (2020). Accessed: June 1, 2020 : https://www.who.int/publications/i/item/clinical-management-of-covid-19.

5. Ranieri V, Rubenfeld G, Thompson B, et al.: Acute respiratory distress syndrome: the Berlin Definition . JAMA. 2012, 307:2526-2533. 10.1001/jama.2012.5669

6. von Elm E, Altman DG, Egger M, Pocock SI, Gøtzsche PC, Vandenbroucke JP, for the STROBE Initiative: The Strengthening the Reporting of Observational Studies in Epidemiology (STROBE) statement: guidelines for reporting observational studies. J Clin Epidemiol. 2008, 61:344-349. 10.1016/j.jclinepi.2007.11.008

7. Tibshirani R: Regression shrinkage and selection via the Lasso. J R Stat Soc Series B Stat Methodol. 1996, 58:267-288. 10.1111/i.2517-6161.1996.tb02080.x

8. Ma Y, Diao B, Lv X, et al.: Epidemiological, clinical, and immunological features of a cluster of COVID-19contracted hemodialysis patients. Kidney Int Rep. 2020, 5:1333-1341. 10.1016/j.ekir.2020.06.003

9. Goicoechea M, Sánchez Cámara LA, Macías N, et al.: COVID-19: clinical course and outcomes of 36 hemodialysis patients in Spain. Kidney Int. 2020, 98:27-34. 10.1016/j.kint.2020.04.031

10. Valeri AM, Robbins-Juarez SY, Stevens IS, et al.: Presentation and outcomes of patients with ESKD and COVID-19. J Am Soc Nephrol. 2020, 31:1409-1415. 10.1681/ASN.2020040470

11. ICNARC report on COVID-19 in critical care . (2020). Accessed: May 21, 2020: https://www.icnarc.org/OurAudit/Audits/Cmp/Reports.

12. Docherty AB, Harrison EM, Green CA, et al.: Features of 20133 UK patients in hospital with covid-19 using the ISARIC WHO Clinical Characterisation Protocol: prospective observational cohort study. BMJ. 2020, 369:m1985. 10.1136/bmi.m1985

13. Wadhera RK, Wadhera P, Gaba P, Figueroa JF, Maddox KEJ, Yeh RW, Shen C: Variation in COVID-19 hospitalizations and deaths across New York City boroughs. JAMA. 2020, 323:2192-2195.

10.1001/jama.2020.7197 
14. U.S. Census Bureau - QuickFacts, New Rochelle city, New York . (2010). Accessed: September 1, 2020: https://www.census.gov/quickfacts/newrochellecitynewyork.

15. Liu Q, Zhou Y-h, Yang Z-q: The cytokine storm of severe influenza and development of immunomodulatory therapy. Cell Mol Immunol. 2016, 13:3-10. 10.1038/cmi.2015.74

16. Channappanavar R, Fett C, Mack M, Ten Eyck PP, Meyerholz DK, Perlman S: Sex based differences in susceptibility to severe acute respiratory syndrome coronavirus infection. J Immunol. 2017, 198:4046-4053. 10.4049/jimmunol.1601896

17. Mehta P, McAuley DF, Brown M, Sanchez E, Tattersall RS, Manson JJ: COVID-19: consider cytokine storm syndromes and immunosuppression. Lancet. 2020, 395:1033-1034. 10.1016/S0140-6736(20)30628-0

18. Chiappetta S, Sharma AM, Bottino V, Stier C: COVID-19 and the role of chronic inflammation in patients with obesity. Int J Obes (Lond). 2020, 44:1790-1792. 10.1038/s41366-020-0597-4

19. Vaziri ND, Pahl MV, Crum A, Norris K: Effect of uremia on structure and function of immune system . J Ren Nutr. 2012, 22:149-156. 10.1053/j.jrn.2011.10.020

20. Stenvinkel P, Alvestrand A: Inflammation in end-stage renal disease: sources, consequences, and therapy . Semin Dial. 2002, 15:329-337. 10.1046/j.1525-139X.2002.00083.x

21. Jofré R, Rodriguez-Benitez P, López-Gómez JM, Pérez-Garcia R: Inflammatory syndrome in patients on hemodialysis. J Am Soc Nephrol. 2006, 17:S274-S280. 10.1681/ASN.2006080926

22. Yao Q, Axelsson J, Stenvinkel P, Lindholm B: Chronic systemic inflammation in dialysis patients: an update on causes and consequences. ASAIO J. 2004, 50:lii-lvii. 10.1097/01.mat.0000147958.87989.eb

23. Sakr Y, Elia C, Mascia L, et al.: Being overweight or obese is associated with decreased mortality in critically ill patients: a retrospective analysis of a large regional Italian multicenter cohort. J Crit Care. 2012, 27:714721. 10.1016/j.jcrc.2012.08.013

24. Acharya P, Upadhyay L, Qavi A, Naaraayan A, Jesmajian S, Acharya S, Bharati R: The paradox prevails: outcomes are better in critically ill obese patients regardless of the comorbidity burden. J Crit Care. 2019, 53:25-31. 10.1016/j.jcrc.2019.05.004

25. Honore PM, Hoste E, Molnár Z, Jacobs R, Joannes-Boyau O, Malbrain MG, Forni LG: Cytokine removal in human septic shock: where are we and where are we going?. Ann Intensive Care. 2019, 9:56. 10.1186/s13613019-0530-y

26. Zhang P, Zhu L, Cai J, et al.: Association of inpatient use of angiotensin converting enzyme inhibitors and angiotensin II receptor blockers with mortality among patients with hypertension hospitalized with COVID19. Circ Res. 2020, 126:1671-1681. 10.1161/CIRCRESAHA.120.317134

27. Malik U, Raizada V: Some aspects of the renin-angiotensin-system in hemodialysis patients . Kidney Blood Press Res. 2015, 40:614-622. 10.1159/000368537

28. Urushihara M, Kobori H: Intrarenal renin-angiotensin system activation in end-stage renal disease . Hypertens Res. 2017, 40:351-352. 10.1038/hr.2017.7

29. Kalantar-Zadeh K, Block G, Humphreys MH, Kopple JD: Reverse epidemiology of cardiovascular risk factors in maintenance dialysis patients. Kidney Int. 2003, 63:793-808. 10.1046/j.1523-1755.2003.00803.x

30. Beddhu S, Abraham J: Risk factor paradox in CKD and ESRD: does a healthy lifestyle matter? . Clin J Am Soc Nephrol. 2013, 8:515-517. 10.2215/CJN.02030213 